\title{
ON MOCANU-TYPE FUNCTIONS WITH GENERALIZED BOUNDED VARIATIONS
}

\section{SHUJAAT ALI SHAH*, MUHAMMAD AFZAL SOOMRO AND ASGHAR ALI MAITLO}

Department of Mathematics and Statistics, Quaid-i-Awam University of Engineering, Science and Technology, Nawabshah, 67480 Sindh, Pakistan

*Corresponding author: shahglike@yahoo.com

Abstract. The main focus of this article is the study of classes $M_{\mu}^{\delta}(\varphi, \mathcal{H})$ and $\mathcal{Q}_{\mu}^{\delta}\left(\varphi, g_{1}, \mathcal{H}\right)$. We present various inclusion relationships and some applications of our investigations are considered. Also, we include radius problem.

\section{Introduction}

Let $\mathcal{A}$ be the class of analytic functions of the form

$$
f(z)=z+\sum_{n=2}^{\infty} a_{n} z^{n},
$$

in the open unit disk $\mathcal{U}=\{z:|z|<1\}$. If $f$ and $g$ are analytic in $\mathcal{U}$, we say that $f$ is subordinate to $g$, written $f \prec g$ or $f(z) \prec g(z)$, if there exists a Schwartz function $w$ in $\mathcal{U}$ such that $f(z)=g(w(z))$.

The convolution or Hadamard product of two functions $f, g \in \mathcal{A}$ is denoted by $f * g$ and is defined as

$$
(f * g)(z)=z+\sum_{n=2}^{\infty} a_{n} b_{n} z^{n}, \quad z \in \mathcal{U}
$$

Analytic functions $p$ in the class $\mathcal{P}[A, B]$ can be defined by using subordination as follows [3].

Let $p$ be analytic in $\mathcal{U}$ with $p(0)=1$. Then $p \in \mathcal{P}[A, B]$, if and only if,

$$
p(z) \prec \frac{1+A z}{1+B z}, \quad-1 \leq B<A \leq 1, z \in \mathcal{U} .
$$

Received July $12^{\text {th }}, 2021$; accepted August 17th 2021 ; published September $14^{\text {th }}, 2021$.

2010 Mathematics Subject Classification. 30C45, 30C55.

Key words and phrases. analytic functions; Janowski functions; conic regions; bounded boundary rotations.

(C)2021 Authors retain the copyrights of their papers, and all open access articles are distributed under the terms of the Creative Commons Attribution License. 
For $k \geqslant 0$, the conic domains $\Omega_{k}$, defined as;

$$
\Omega_{k}=\left\{u+i v: u>k \sqrt{(u-1)^{2}+v^{2}}\right\} .
$$

The domains $\Omega_{k}(k=0)$ represents right half plane, $\Omega_{k}(0<k<1)$ represents hyperbola, $\Omega_{k}(k=1)$ represents a parabola and $\Omega_{k}(k>1)$ represents an ellipse. The extremal functions for these conic regions are given as;

$$
p_{k}(z)=\left\{\begin{array}{lr}
\frac{1+z}{1-z}, & k=0 \\
1+\frac{2}{\pi^{2}}\left(\log \frac{1+\sqrt{z}}{1-\sqrt{z}}\right)^{2}, & k=1 \\
1+\frac{2}{1-k^{2}}\left[\left(\frac{2}{\pi} \arccos k\right) \arctan h \sqrt{z}\right], & 0<k<1 \\
1+\frac{1}{k^{2}-1} \sin \left(\frac{\pi}{2 R(t)} \int_{0}^{\frac{u(z)}{\sqrt{t}}} \frac{1}{\sqrt{1-x^{2}} \sqrt{1-(t x)^{2}}} d x\right)+\frac{1}{k^{2}-1}, k>1,
\end{array}\right.
$$

where $u(z)=\frac{z-\sqrt{t}}{z-\sqrt{t z}}, t \in(0,1), z \in \mathcal{U}$ and $z$ is chosen such that $k=\cosh \left(\frac{\pi R^{\prime}(t)}{4 R(t)}\right), R(t)$ is Legendre's complete elliptic integral of the first kind and $R^{\prime}(t)$ is complementary integral of $R(t)$. See $[4,5]$ for more information. These conic regions are being studied by several authors, see $[6,9,12]$.

In 2017, Dziok and Noor [2] introduced and studied the concepts of some general classes given as below.

Definition 1.1. Let $\mu \geq 0, \Phi=\left(\phi_{1}(z), \phi_{2}(z)\right)$ and $\mathcal{H}=\left(h_{1}(z), h_{2}(z)\right)$ where $h_{i} \in \mathcal{A}$ with $h_{i}(0)=1$, $(i=1,2)$. Then

$$
\mathcal{P}_{\mu}(\mathcal{H})=\left\{\mu q_{1}+(1-\mu) q_{2}: q_{1} \in \mathcal{P}\left(h_{1}\right), q_{2} \in \mathcal{P}\left(h_{2}\right)\right\}
$$

where

$$
\mathcal{P}(h)=\{q \in \mathcal{A}: q \prec h \text { with } q(0)=1\}
$$

Some special cases:

(i) $\mathcal{P}_{\mu}(h)=\mathcal{P}_{\mu}((h, h))$. If $\mu=\frac{m}{4}+\frac{1}{2},(m \geq 2)$, then $\mathcal{P}_{\mu}(h)=\mathcal{P}_{m}(h)$.

(ii) If $\mu=\frac{m}{4}+\frac{1}{2},(m \geq 2)$, and $h(z)=\frac{1+(1-2 \rho) z}{1-z}$, then $\mathcal{P}_{\mu}(h)=\mathcal{P}_{m}(\rho)$, this class was introduced by Padmanabhan et al. [13].

(iii) If $\mu=\frac{m}{4}+\frac{1}{2},(m \geq 2)$ and $h(z)=\frac{1+A z}{1+B z}(-1 \leq B<A \leq 1)$, then $\mathcal{P}_{\mu}(h)=\mathcal{P}_{m}[A, B]$, this class was introduced by Noor [10]. Moreover, for $A=1$ and $B=-1$ we have $\mathcal{P}_{\mu}(h)=\mathcal{P}_{m}$; see [14].

(iv) If $\mu=\frac{m}{4}+\frac{1}{2},(m \geq 2)$ and $h(z)=p_{\kappa}(z)(\kappa \geq 0)$, then $\mathcal{P}_{\mu}(h)=\mathcal{P}_{m}\left(p_{\kappa}\right)$, this class was defined by Noor et al. [11].

Definition 1.2. Let $f \in \mathcal{A}$ and $\delta \geq 0$. Then $f \in M_{\mu}^{\delta}(\Phi, \xi, \mathcal{H})$ if and only if $J_{\delta}(f((z))) \in \mathcal{P}_{\mu}(\mathcal{H})$, where

$$
J_{\delta}(f((z)))=(1-\delta) \frac{\left(\xi * \phi_{2}\right) * f}{\left(\xi * \phi_{1}\right) * f}+\delta \frac{\phi_{2} * f}{\phi_{1} * f} .
$$

If $\xi_{1}(z)=z+\sum_{n=2}^{\infty} \frac{1}{n} z^{n}, \phi_{1}(z)=z \varphi^{\prime}(z)$ and $\phi_{2}(z)=z \phi_{1}^{\prime}(z)$, then we have the following special cases.

$$
M^{\delta}(\Phi, \xi, h)=M_{1}^{\delta}(\Phi, \xi,(h, h)), M_{\mu}^{\delta}(\Phi, \mathcal{H})=M_{\mu}^{\delta}\left(\Phi, \xi_{1}, \mathcal{H}\right),
$$




$$
\begin{gathered}
M_{\mu}^{\delta}(\varphi, \mathcal{H})=M_{\mu}^{\delta}\left(\left(\phi_{2}, \phi_{1}\right), \mathcal{H}\right), \\
S_{\mu}^{*}(\varphi, \mathcal{H})=M_{\mu}^{0}(\varphi, \mathcal{H}), \quad S^{*}(\varphi, h)=M_{1}^{0}(\varphi, h) .
\end{gathered}
$$

Definition 1.3. Let $f \in \mathcal{A}, \mathcal{G}=\left(g_{1}, g_{2}\right)$, where $g_{i} \in \mathcal{A}$ with $g_{i}(0)=1(i=1,2)$, and $\delta, \vartheta \geq 0$. Then $f \in \mathcal{Q}_{\mu, \vartheta}^{\delta}(\Phi, \xi, \mathcal{G}, \mathcal{H})$ if there exists $g \in S_{\vartheta}^{*}(\varphi, \mathcal{G})$ such that

$$
(1-\delta) \frac{\left(\xi * \phi_{2}\right) * f}{\left(\xi * \phi_{1}\right) * g}+\delta \frac{\phi_{2} * f}{\phi_{1} * g} \in \mathcal{P}_{\mu}(\mathcal{H}) .
$$

If $\xi_{1}(z)=z+\sum_{n=2}^{\infty} \frac{1}{n} z^{n}, \phi_{1}(z)=z \varphi^{\prime}(z)$ and $\phi_{2}(z)=z \phi_{1}^{\prime}(z)$, then we have the following special cases.

$$
\begin{gathered}
\mathcal{Q}^{\delta}\left(\Phi, \xi, g_{1}, h_{1}\right)=M_{1,1}^{\delta}\left(\Phi, \xi,\left(g_{1}, g_{2}\right),\left(h_{1}, h_{2}\right)\right) \\
\mathcal{Q}_{\mu, \vartheta}^{\delta}(\Phi, \mathcal{G}, \mathcal{H})=M_{\mu, \vartheta}^{\delta}\left(\Phi, \xi_{1}, \mathcal{G}, \mathcal{H}\right) \\
\mathcal{Q}_{\mu}^{\delta}\left(\varphi, g_{1}, H\right)=\mathcal{Q}_{\mu, 1}^{\delta}\left(\left(\phi_{2}, \phi_{1}\right),\left(g_{1}, g_{1}\right), H\right)
\end{gathered}
$$

From (1.4), we denote the class $M_{\mu}^{\delta}(\varphi, \mathcal{H})$ of functions $f \in \mathcal{A}$ satisfies $J_{\delta}(f(z)) \in \mathcal{P}_{\mu}(\mathcal{H})$, where

$$
J_{\delta}(f(z))=(1-\delta) \frac{z(\varphi * f)^{\prime}}{(\varphi * f)}+\delta \frac{\left(z(\varphi * f)^{\prime}\right)^{\prime}}{(\varphi * f)^{\prime}}
$$

and $\mathcal{P}_{\mu}(\mathcal{H})$ is given by Definition 1.1 .

Similarly, from (1.6), we denote the class $\mathcal{Q}_{\mu}^{\delta}(\varphi, h, \mathcal{H})$ of functions $f \in \mathcal{A}$ satisfies $J_{\delta}(f(z), g(z)) \in \mathcal{P}_{\mu}(\mathcal{H})$, where

$$
J_{\delta}(f(z), g(z))=(1-\delta) \frac{z(\varphi * f)^{\prime}}{(\varphi * g)}+\delta \frac{\left(z(\varphi * f)^{\prime}\right)^{\prime}}{(\varphi * g)^{\prime}},
$$

for $g \in S^{*}(\varphi, h)$, the class $S^{*}(\varphi, h)$ is given by (1.5).

\section{Preliminary Results}

Lemma 2.1. [2] Let $\mathcal{H}=\left(h_{1}, h_{2}\right)$, where $h_{i}(i=1,2)$ are analytic, univalent convex functions with $h_{i}(0)=1$ $(i=1,2)$ and let $\varkappa: U \rightarrow \mathbb{C}$ (set of complex numbers) with $\Re(\varkappa)>0$. If $p(z)$ is analytic, with $p(0)=1$ in $\mathcal{U}$, satisfies

$$
p(z)+\varkappa z p^{\prime}(z) \in \mathcal{P}_{\mu}(\mathcal{H}),
$$

then $p(z) \in \mathcal{P}_{\mu}(\mathcal{H})$. 
Lemma 2.2. [8] Let $h$ be analytic, univalent convex function in $\mathcal{U}$ with $h(0)=1$ and $\operatorname{Re}(\gamma h(z)+\sigma)>0$, $\sigma, \gamma \in \mathbb{C}$ and $\gamma \neq 0$. If $p(z)$ is analytic in $\mathcal{U}$ and $p(0)=h(0)$, then

$$
\left\{p(z)+\frac{z p^{\prime}(z)}{\gamma p(z)+\sigma}\right\} \prec h(z)
$$

implies $p(z) \prec q(z) \prec h(z)$, where $q(z)$ is best dominant and is given as,

$$
q(z)=\left[\left\{\int_{0}^{1}\left(\exp \int_{t}^{t z} \frac{h(u)-1}{u} d u\right) d t\right\}^{-1}-\frac{\sigma}{\gamma}\right] .
$$

Lemma 2.3. [15] If $f \in C, g \in S^{*}$, then for each $h$ analytic in $\mathcal{U}$ with $h(0)=1$,

$$
\frac{(f * h g)(\mathcal{U})}{(f * g)(\mathcal{U})} \subset \overline{C o} h(\mathcal{U})
$$

where $\overline{C o} h(\mathcal{U})$ denotes the convex hull of $h(\mathcal{U})$.

\section{MAin Results}

\subsection{Inclusion Results.}

Theorem 3.1. Let $\delta \geq 0, \varphi \in \mathcal{A}$ and $h$ be any convex univalent function in $\mathcal{U}$. Then

$$
M_{1}^{\delta}(\varphi, h) \subset M_{1}^{0}(\varphi, h) .
$$

Proof. Let $f \in M_{1}^{\delta}(\varphi, h)$. Then, by definition,

$$
(1-\delta) \frac{z(\varphi * f)^{\prime}}{(\varphi * f)}+\delta \frac{\left(z(\varphi * f)^{\prime}\right)^{\prime}}{(\varphi * f)^{\prime}} \in \mathcal{P}(h),
$$

or

$$
(1-\delta) \frac{z(\varphi * f)^{\prime}}{(\varphi * f)}+\delta \frac{\left(z(\varphi * f)^{\prime}\right)^{\prime}}{(\varphi * f)^{\prime}} \prec h(z) .
$$

Consider

$$
\frac{z(\varphi * f)^{\prime}}{(\varphi * f)}=p(z)
$$

On logarithmic differentiation of (3.2), we have

$$
\frac{\left(z(\varphi * f)^{\prime}\right)^{\prime}}{(\varphi * f)^{\prime}}=\frac{z(\varphi * f)^{\prime}}{(\varphi * f)}+\frac{z p^{\prime}(z)}{p(z)} .
$$

From (3.2) and (3.3), we get

$$
\frac{\left(z(\varphi * f)^{\prime}\right)^{\prime}}{(\varphi * f)^{\prime}}=p(z)+\frac{z p^{\prime}(z)}{p(z)} .
$$

On making use of (3.2) and (3.4) in (3.1), we obtain

$$
(1-\delta) p(z)+\delta\left[p(z)+\frac{z p^{\prime}(z)}{p(z)}\right] \prec h(z)
$$

this implies

$$
p(z)+\delta \frac{z p^{\prime}(z)}{p(z)} \prec h(z) .
$$

By using Lemma 2.2, we conclude $p(z) \prec h(z)$. Hence $f \in M_{1}^{0}(\varphi, h)$. 
Remark 3.1. Following different choices of $\varphi$ and $h$ give certain inclusion results for the above theorem.

(i) $\varphi \in A, h(z)=\frac{1+A z}{1+B z}$, where $-1 \leq B<A \leq 1$.

(ii) $\varphi \in A, h(z)=p_{k}(z)$, where $p_{k}(z)$ is given by (1.3).

Corollary 3.1. Let $\delta \geq 1$. Then

$$
M_{1}^{\delta}(\varphi, h) \subset M_{1}^{1}(\varphi, h) .
$$

Proof. Let $f \in M_{1}^{\delta}(\varphi, h)$. Then, by definition,

$$
(1-\delta) \frac{z(\varphi * f)^{\prime}}{(\varphi * f)}+\delta \frac{\left(z(\varphi * f)^{\prime}\right)^{\prime}}{(\varphi * f)^{\prime}}=s_{1}(z) \prec h(z),
$$

from previous theorem, we can write

$$
\frac{z(\varphi * f)^{\prime}}{(\varphi * f)}=s_{2}(z) \prec h(z)
$$

Now,

$$
\begin{aligned}
\delta \frac{\left(z(\varphi * f)^{\prime}\right)^{\prime}}{(\varphi * f)^{\prime}} & =\left[(1-\delta) \frac{z(\varphi * f)^{\prime}}{(\varphi * f)}+\delta \frac{\left(z(\varphi * f)^{\prime}\right)^{\prime}}{(\varphi * f)^{\prime}}\right]+(\delta-1) \frac{z(\varphi * f)^{\prime}}{(\varphi * f)} \\
& =s_{1}(z)+(\delta-1) s_{2}(z) .
\end{aligned}
$$

Implies that

$$
\frac{\left(z(\varphi * f)^{\prime}\right)^{\prime}}{(\varphi * f)^{\prime}}=\left(1-\frac{1}{\delta}\right) s_{2}(z)+\frac{1}{\delta} s_{1}(z) .
$$

Since $s_{1}, s_{2} \prec h(z),(3.5)$ gives us

$$
\frac{\left(z(\varphi * f)^{\prime}\right)^{\prime}}{(\varphi * f)^{\prime}} \prec h(z) .
$$

Hence $f \in M_{1}^{\delta}(\varphi, h)$.

Remark 3.2. The different choices of $\varphi$ and h given in Remark 3.1 hold the inclusion result proved in above theorem.

Theorem 3.2. Let $\delta, \mu \geq 0, \varphi \in \mathcal{A}, \mathcal{H}=\left(h_{1}, h_{2}\right)$ where $h_{i}, h \in \mathcal{A}$ with $h_{i}(0)=h(0)=1(i=1,2)$. Then

$$
\mathcal{Q}_{\mu}^{\delta}(\varphi, h, \mathcal{H}) \subset \mathcal{Q}_{\mu}^{0}(\varphi, h, \mathcal{H})
$$

Proof. Let $f \in \mathcal{Q}_{\mu}^{\delta}(\varphi, h, \mathcal{H})$. Then, by definition,

$$
(1-\delta) \frac{z(\varphi * f)^{\prime}}{(\varphi * g)}+\delta \frac{\left(z(\varphi * f)^{\prime}\right)^{\prime}}{(\varphi * g)^{\prime}} \in \mathcal{P}_{\mu}(\mathcal{H}),
$$

for $g \in S^{*}(\varphi, h)$.

Consider

$$
\frac{z(\varphi * f)^{\prime}}{(\varphi * g)}=p(z)
$$


where $p(z)$ is analytic with $p(0)=1$ in $\mathcal{U}$.

On logarithmic differentiation of (3.7), we get

$$
\begin{gathered}
\frac{\left(z(\varphi * f)^{\prime}\right)^{\prime}}{(\varphi * f)^{\prime}}=\frac{z(\varphi * g)^{\prime}}{(\varphi * g)}+\frac{z p^{\prime}(z)}{p(z)}, \\
\frac{\left(z(\varphi * f)^{\prime}\right)^{\prime}}{(\varphi * g)^{\prime}}=\frac{z(\varphi * f)^{\prime}}{(\varphi * g)^{\prime}}\left[\frac{z(\varphi * g)^{\prime}}{(\varphi * g)}+\frac{z p^{\prime}(z)}{\frac{z(\varphi * f)^{\prime}}{(\varphi * g)}}\right],
\end{gathered}
$$

this implies

$$
\frac{\left(z(\varphi * f)^{\prime}\right)^{\prime}}{(\varphi * g)^{\prime}}=\frac{z(\varphi * f)^{\prime}}{(\varphi * g)}+\frac{z p^{\prime}(z)}{\frac{z(\varphi * g)^{\prime}}{(\varphi * g)}}
$$

From (3.7) and (3.8), we have

$$
\frac{\left(z(\varphi * f)^{\prime}\right)^{\prime}}{(\varphi * g)^{\prime}}=p(z)+\frac{z p^{\prime}(z)}{p_{0}(z)} ; \text { with } p_{0}(z)=\frac{z(\varphi * g)^{\prime}}{(\varphi * g)} .
$$

Now, from (3.6), (3.7) and (3.9), we obtain

$$
(1-\delta) p(z)+\delta\left(p(z)+\frac{z p^{\prime}(z)}{p_{0}(z)}\right) \in \mathcal{P}_{\mu}(\mathcal{H}),
$$

or equivalently,

$$
p(z)+\frac{\delta}{p_{0}(z)} z p^{\prime}(z) \in \mathcal{P}_{\mu}(\mathcal{H}) .
$$

If $g \in S^{*}(\varphi, h)$, then $\frac{z(\varphi * g)^{\prime}}{(\varphi * g)} \prec h(z) ; h \in \mathcal{P}$. This implies $\Re\left(p_{0}(z)\right)>0$ in $\mathcal{U}$. Thus, by Lemma 2.1 , we conclude $p(z) \in \mathcal{P}_{\mu}(\mathcal{H})$. Consequently, $\frac{z(\varphi * f)^{\prime}}{(\varphi * g)} \in \mathcal{P}_{\mu}(\mathcal{H})$. Hence, $f \in \mathcal{Q}_{\mu}^{0}(\varphi, h, \mathcal{H})$.

Remark 3.3. It is easy to see that the inclusion in Theorem 3.2 is true for different choices of $\varphi$, $h$ and $\mathcal{H}=\left(h_{1}, h_{2}\right)$ given as following.

(i) $\varphi \in A, h_{1}(z)=\frac{1+A z}{1+B z}=h_{2}(z)$, where $-1 \leq B<A \leq 1$.

(ii) $\varphi \in A, h_{1}(z)=p_{k}(z)=h_{2}(z)$, where $p_{k}(z)$ is given by (1.3).

(iii) $\varphi \in A, h_{1}(z)=\frac{1+A z}{1+B z}, \quad h_{2}(z)=p_{k}(z)$.

(iv) $\varphi \in A, h_{1}(z)=p_{k}(z), h_{2}(z)=\frac{1+A z}{1+B z}$.

Corollary 3.2. Let $\delta \geq 1$. Then

$$
\mathcal{Q}_{\mu}^{\delta}(\varphi, h, \mathcal{H}) \subset \mathcal{Q}_{\mu}^{1}(\varphi, h, \mathcal{H})
$$

Proof. Let $f \in \mathcal{Q}_{\mu}^{\delta}(\varphi, h, \mathcal{H})$. Then, by definition,

$$
(1-\delta) \frac{z(\varphi * f)^{\prime}}{(\varphi * g)}+\delta \frac{\left(z(\varphi * f)^{\prime}\right)^{\prime}}{(\varphi * g)^{\prime}}=p_{1}(z) \in \mathcal{P}_{\mu}(\mathcal{H}),
$$

where $g \in S^{*}(\varphi, h)$.

From previous theorem, we can write

$$
\frac{z(\varphi * f)^{\prime}}{(\varphi * g)}=p_{2}(z) \in \mathcal{P}_{\mu}(\mathcal{H})
$$


Now,

$$
\begin{aligned}
\delta \frac{\left(z(\varphi * f)^{\prime}\right)^{\prime}}{(\varphi * g)^{\prime}} & =\left[(1-\delta) \frac{z(\varphi * f)^{\prime}}{(\varphi * g)}+\delta \frac{\left(z(\varphi * f)^{\prime}\right)^{\prime}}{(\varphi * g)^{\prime}}\right]+(\delta-1) \frac{z(\varphi * f)^{\prime}}{(\varphi * g)} \\
& =p_{1}(z)+(\delta-1) p_{2}(z) .
\end{aligned}
$$

This implies

$$
\frac{\left(z(\varphi * f)^{\prime}\right)^{\prime}}{(\varphi * g)^{\prime}}=\left(1-\frac{1}{\delta}\right) p_{2}(z)+\frac{1}{\delta} p_{1}(z) .
$$

Since $p_{1}, p_{2} \in \mathcal{P}_{\mu}(\mathcal{H})$ and $\mathcal{P}_{\mu}(\mathcal{H})$ is convex set, then

$$
\frac{\left(z(\varphi * f)^{\prime}\right)^{\prime}}{(\varphi * g)^{\prime}} \in \mathcal{P}_{\mu}(\mathcal{H}) .
$$

Hence $f \in \mathcal{Q}_{\mu}^{1}(\varphi, h, \mathcal{H})$.

Theorem 3.3. Let $0 \leq \delta_{1}<\delta$. Then

$$
\mathcal{Q}_{\mu}^{\delta}(\varphi, h, \mathcal{H}) \subset \mathcal{Q}_{\mu}^{\delta_{1}}(\varphi, h, \mathcal{H})
$$

Proof. If $\delta_{1}=0$, then it is obvious from Theorem 3.2.

For $\delta_{1}>0$. Let $f \in \mathcal{Q}_{\mu}^{\delta}(\varphi, h, H)$. Then, from Theorem 3.2

$$
\frac{z(\varphi * f)^{\prime}}{(\varphi * g)}=p_{2}(z) \in \mathcal{P}_{\mu}(\mathcal{H})
$$

As we can write

$$
\begin{aligned}
& \left(1-\delta_{1}\right) \frac{z(\varphi * f)^{\prime}}{(\varphi * g)}+\delta_{1} \frac{\left(z(\varphi * f)^{\prime}\right)^{\prime}}{(\varphi * g)^{\prime}} \\
= & \frac{\delta_{1}}{\delta}\left[\left(\frac{\delta}{\delta_{1}}-1\right) \frac{z(\varphi * f)^{\prime}}{(\varphi * g)}+(1-\delta) \frac{z(\varphi * f)^{\prime}}{(\varphi * g)}+\delta \frac{\left(z(\varphi * f)^{\prime}\right)^{\prime}}{(\varphi * g)^{\prime}}\right] .
\end{aligned}
$$

Since $f \in \mathcal{Q}_{\mu}^{\delta}(\varphi, h, \mathcal{H})$, from definition of $\mathcal{Q}_{\mu}^{\delta}(\varphi, h, \mathcal{H})$, we have

$$
(1-\delta) \frac{z(\varphi * f)^{\prime}}{(\varphi * g)}+\delta \frac{\left(z(\varphi * f)^{\prime}\right)^{\prime}}{(\varphi * g)^{\prime}}=p_{1}(z) \in \mathcal{P}_{\mu}(\mathcal{H})
$$

From (3.10-3.12) and the convexity of $\mathcal{P}_{\mu}(\mathcal{H})$ implies

$$
\left(1-\delta_{1}\right) \frac{z(\varphi * f)^{\prime}}{(\varphi * g)}+\delta_{1} \frac{\left(z(\varphi * f)^{\prime}\right)^{\prime}}{(\varphi * g)^{\prime}} \in \mathcal{P}_{\mu}(\mathcal{H}) .
$$

Hence $f \in \mathcal{Q}_{\mu}^{\delta_{1}}(\varphi, h, \mathcal{H})$.

Remark 3.4. It is easy to see that the inclusion in Theorem 3.3 is true for all choices given in Remark 3.3.

Theorem 3.4. The class $\mathcal{Q}_{\mu}^{\delta}(\varphi, h, \mathcal{H})$ is closed under the convex convolution. 
Proof. Let $f \in \mathcal{Q}_{\mu}^{\delta}(\varphi, h, \mathcal{H})$. Then, by definition,

$$
(1-\delta) \frac{z(\varphi * f)^{\prime}}{(\varphi * g)}+\delta \frac{\left(z(\varphi * f)^{\prime}\right)^{\prime}}{(\varphi * g)^{\prime}} \in \mathcal{P}_{\mu}(\mathcal{H}) .
$$

First, we need to prove $\varsigma * f \in \mathcal{Q}_{\mu}^{0}(\varphi, h, \mathcal{H})$ for $\varsigma \in C$.

We take $\delta=0$, then (3.13) implies

$$
\frac{z(\varphi * f)^{\prime}}{(\varphi * g)} \in \mathcal{P}_{\mu}(\mathcal{H})
$$

Let

$$
\begin{aligned}
\frac{z(\varphi *(\varsigma * f))^{\prime}(z)}{(\varphi *(\varsigma * g))(z)} & =\frac{\varsigma * \frac{z(\varphi * f)^{\prime}}{(\varphi * g)}((\varphi * g))(z)}{\varsigma *(\varphi * g)(z)} \\
& =\frac{\varsigma * h_{0}(z)((\varphi * g))(z)}{\varsigma *(\varphi * g)(z)}
\end{aligned}
$$

where $h_{0}(z)=\frac{z(\varphi * f)^{\prime}}{(\varphi * g)} \in \mathcal{P}_{\mu}(\mathcal{H})$. Since $g \in S^{*}(\varphi, h)$ implies $\varphi * g \in S^{*}(h) \subset S^{*} ; h \in \mathcal{P}$. Thus, by Lemma 2.3 , we conclude

$$
\frac{z(\varphi *(\varsigma * f))^{\prime}(z)}{(\varphi *(\varsigma * g))(z)} \in \mathcal{P}_{\mu}(\mathcal{H})
$$

Similarly, for $\delta=1$, we can easily prove

$$
\frac{z\left(\varphi *(\varsigma * f)^{\prime}\right)^{\prime}(z)}{(\varphi *(\varsigma * g))^{\prime}(z)} \in \mathcal{P}_{\mu}(\mathcal{H}) .
$$

Our required result follows from (3.15) and (3.16).

Corollary 3.3. The class $\mathcal{Q}_{\mu}^{\delta}(\varphi, h, \mathcal{H})$ is closed under the following operators.

(i) $f_{1}(z)=\int_{0}^{z} \frac{f(t)}{t} d t$.

(ii) $f_{2}(z)=\frac{2}{z} \int_{0}^{z} f(t) d t, \quad$ (Libera's operator [7]).

(iii) $f_{3}(z)=\int_{0}^{z} \frac{f(t)-f(x t)}{t-x t} d t, \quad|x| \leq 1, x \neq 1$.

(iv) $f_{4}(z)=\frac{c+1}{z^{c}} \int_{0}^{z} t^{c-1} f(t), \quad \operatorname{Re}(c) \geq 0, \quad$ (Generalized Bernardi operator [1]).

Proof. We may write, $f_{i}(z)=f(z) * \phi_{i}(z)$, where $\phi_{i}(z), i=1,2,3,4$, are convex and given by

$$
\begin{aligned}
& \phi_{1}(z)=-\log (1-z)=\sum_{n=1}^{\infty} \frac{1}{n} z^{n}, \\
& \phi_{2}(z)=\frac{-2[z-\log (1-z)]}{z}=\sum_{n=1}^{\infty} \frac{2}{n+1} z^{n}, \\
& \phi_{3}(z)=\frac{1}{1-x} \log \left(\frac{1-x z}{1-z}\right)=\sum_{n=1}^{\infty} \frac{1-x^{n}}{(1-x)^{n}} z^{n}, \quad|x| \leq 1, x \neq 1, \\
& \phi_{4}(z)=\sum_{n=1}^{\infty} \frac{1+c}{n+c} z^{n}, \quad \operatorname{Re}(c) \geq 0 .
\end{aligned}
$$

The proof follows easily by using Theorem 3.4. 


\subsection{Radius Problem.}

Theorem 3.5. Let $f \in M_{1}^{0}\left(\varphi, \frac{1+A z}{1+B z}\right)$. Then, $f \in M_{1}^{\delta}\left(\varphi, \frac{1+z}{1-z}\right)$ for $|z|<r_{\delta}$, where

$$
r_{\delta}=\frac{2 A^{2}}{\{\delta(A-B)+2 A\}+\sqrt{\delta^{2}(A-B)^{2}+4 A \delta(A-B)}} .
$$

Proof. Let $f \in M_{1}^{0}\left(\varphi, \frac{1+A z}{1+B z}\right)$. Then, by definition,

$$
\frac{z(\varphi * f)^{\prime}}{(\varphi * f)}=p(z) \prec \frac{1+A z}{1+B z}
$$

On logrithmic differentiation of (3.17), we get

$$
\frac{\left(z(\varphi * f)^{\prime}\right)^{\prime}}{(\varphi * f)^{\prime}}=\frac{z(\varphi * f)^{\prime}}{(\varphi * f)}+\frac{z p^{\prime}(z)}{p(z)}
$$

By (3.17) and (3.18), we obtain

$$
\frac{\left(z(\varphi * f)^{\prime}\right)^{\prime}}{(\varphi * f)^{\prime}}=p(z)+\frac{z p^{\prime}(z)}{p(z)}
$$

Now,

$$
\begin{gathered}
(1-\delta) \frac{z(\varphi * f)^{\prime}}{(\varphi * g)}+\delta \frac{\left(z(\varphi * f)^{\prime}\right)^{\prime}}{(\varphi * g)^{\prime}}=p(z)+\delta \frac{z p^{\prime}(z)}{p(z)} . \\
\Re\left(J_{\delta}(f(z))\right) \geq \frac{A^{2} r^{2}-\{\delta(A-B)+2 A\} r+1}{(1-A r)(1-B r)} .
\end{gathered}
$$

For $\Re\left(J_{\delta}(f(z))\right)>0$ in $\mathcal{U}$, we get

$$
r_{\delta}=\frac{2 A^{2}}{\{\delta(A-B)+2 A\}+\sqrt{\delta^{2}(A-B)^{2}+4 A \delta(A-B)}} .
$$

Corollary 3.4. Let $f \in M_{1}^{0}\left(\frac{z}{1-z}, \frac{1+z}{1-z}\right)=S^{*}$. Then

$$
f \in M_{1}^{\delta}\left(\frac{z}{1-z}, \frac{1+z}{1-z}\right)=M(\delta)
$$

for $|z|<r_{\delta}=\frac{1}{(1+\delta)+\sqrt{\delta^{2}+2 \delta}}$. Moreover, for $\delta=1$, we have well known result

$$
S^{*} \subset C, \text { for }|z|<r_{1}=\frac{1}{2+\sqrt{3}}
$$

Conflicts of Interest: The author(s) declare that there are no conflicts of interest regarding the publication of this paper. 


\section{REFERENCES}

[1] S.D. Bernardi, Convex and starlike univalent functions, Trans. Amer. Math. Soc. 135 (1969), 429-446.

[2] J. Dziok, K.I. Noor, Classes of analytic functions related to a combination of two convex functions, J. Math. Inequal. 11 (2017), 413-427.

[3] W. Janowski, Some extremal problems for certain families of analytic functions I, Ann. Polon. Math. 28 (1973), $297-326$.

[4] S. Kanas, A. Wisniowska, Conic regions and k-uniform convexity, J. Comput. Appl. Math. 105 (1999), $327-336$.

[5] S. Kanas and A. Wisniowska, Conic domain and starlike functions, Rev. Roumaine Math. Pures Appl. 45 (2000), $647-657$.

[6] H.A. Al-Kharsani and A. Sofo, Subordination results on harmonic k-uniformly convex mappings and related classes, Comput. Math. Appl. 59 (2010), 3718-3726.

[7] R.J. Libera, Some classes of regular univalent functions, Proc. Amer. Math. Soc. 16 (1965), 755-758.

[8] S.S. Miller, P.T. Mocanu, Second order differential inequalities in the complex plane, J. Math. Anal. Appl. 65(2) (1978), 289-305.

[9] K.I. Noor, M. Arif, W. Ul-Haq, On k-uniformly close-to-convex functions of complex order, Appl. Math. Comput. 215 (2009), 629-635.

[10] K.I. Noor, Some properties of analytic functions with bounded radius rotations, Compl. Var. Ellipt. Eqn. 54 (2009), $865-877$.

[11] K.I. Noor, M.A. Noor, Higher order close-to-convex functions related with conic domains, Appl. Math. Inf. Sci. 8 (2014), $2455-2463$.

[12] H. Orhan, E. Deniz D. Raducanu, The Fekete-Szego problem for subclasses of analytic functions de ned by a di erential operator related to conic domains, Comput. Math. Appl. 59 (2010), 283-295.

[13] K.S. Padmanabhan, R. Parvatham, Properties of a class of functions with bounded boundary rotation, Ann. Polon. Math. 31 (1975), 311-323.

[14] B. Pinchuk, Functions with bounded boundary rotation, Israel J. Math. 10 (1971), 7-16.

[15] S. Ruscheweyh, T. Sheil-Small, Hadamard product of Schlicht functions and the Polya-Schoenberg conjecture, Comment. Math. Helv. 48 (1973), 119-135. 